\title{
Clinical influences of anticentromere antibody on primary Sjögren's syndrome in a prospective Korean cohort
}

Youngjae Park ${ }^{1}$, Jennifer Lee ${ }^{1}$, Jung Hee Koh², Jung Yoon Choe ${ }^{3}$, Yoon-Kyoung Sung ${ }^{4}$, Shin-Seok Lee ${ }^{5}$, Ji-Min Kim ${ }^{6}$, Sung-Hwan Park ${ }^{1}$, and Seung-Ki Kwok ${ }^{1}$

${ }^{1}$ Division of Rheumatology, Department of Internal Medicine, Seoul St. Mary's Hospital, College of Medicine, The Catholic University of Korea, Seoul; 'Division of Rheumatology, Department of Internal Medicine, Bucheon St. Mary's Hospital, College of Medicine, the Catholic University of Korea, Bucheon; ${ }^{3}$ Division of Rheumatology, Daegu Catholic University Medical Center, Daegu; ${ }^{4}$ Department of Rheumatology, Hanyang University Hospital for Rheumatic Diseases, Seoul; ${ }^{5}$ Department of Rheumatology, Chonnam National University Hospital, Gwangju; ${ }^{6}$ Division of Rheumatology, Department of Internal Medicine, Keimyung University Dongsan Medical Center, Daegu, Korea

\section{Clinical influences of anticentromere antibody on primary Sjögren's syndrome in a prospective Korean cohort}

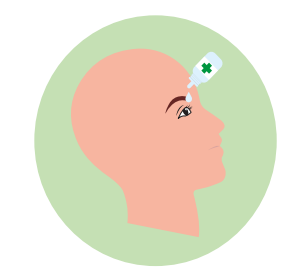

KISS cohort

(Korean nationwide

prospective cohort)

501 primary Sjögren's syndrome

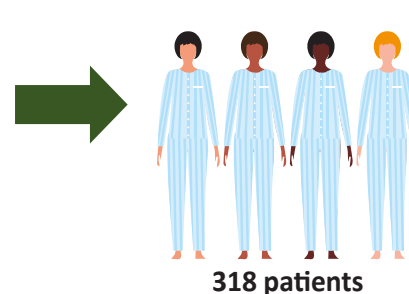

(who fulfilled the 2016 ACR/EULAR classification)
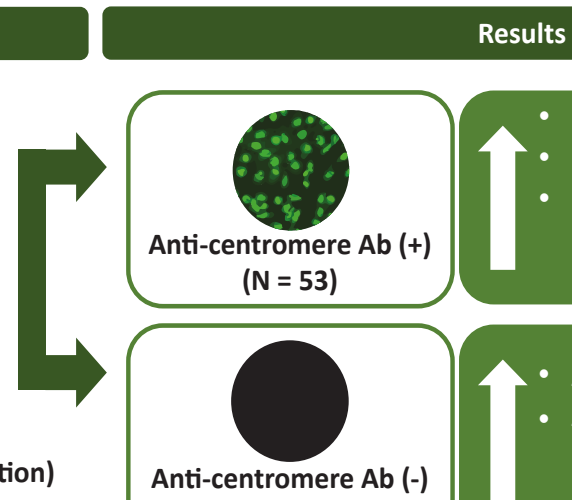

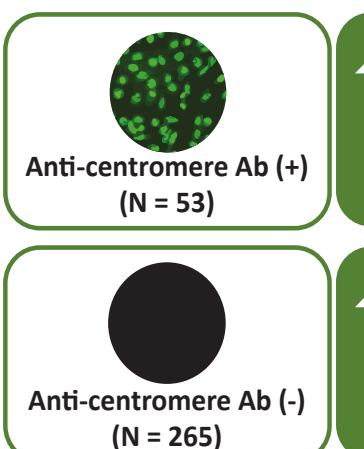

$(\mathrm{N}=\mathbf{2 6 5})$

\section{Conclusion:}

- Anticentromere antibody makes distinguishing influences on clinical phenotypes in primary Sjögren's syndrome.

- Considering distinct phenotypes in hematological and serological features and EGMs, we should monitor the occurrence of these clinical features among pSS patients with ACA in caution.

Received: April 9, 2020 Revised : May 3, 2020 Accepted: May 4, 2020
Correspondence to Seung-Ki Kwok, M.D.

Division of Rheumatology, Department of Internal Medicine, Seoul St. Mary's Hospital, College of Medicine, The Catholic University of Korea, 222 Banpo-daero, Seocho-gu, Seoul 06591, Korea

Tel: +82-2-2258-6014, Fax: +82-2-599-3589, E-mail: seungki73@catholic.ac.kr

https://orcid.org/0000-0002-6142-8364 
Background/Aims: This study was performed to clarify influences of anticentromere antibody (ACA) on clinical phenotypes of primary Sjögren's syndrome (pSS) patients in Korea.

Methods: We assessed 318 patients who met the 2016 American College of Rheumatology/European League Against Rheumatism classification criteria for pSS. All patients were selected from the Korean Initiative of primary Sjögren's Syndrome (KISS), a prospective cohort. Among them, 53 patients were positive for ACA, while another 265 patients were not. We compared various clinical data including demographic features, extra-glandular manifestations (EGMs), clinical indices, and laboratory values available from the KISS database between the two groups.

Results: Patients in the ACA-positive pSS group were older $(p=0.042)$, and had higher xerostomia inventory scores $(p=0.040)$, whereas glandular dysfunction represented with Schirmer I test was more severe in the ACA-negative group. More frequent Raynaud's phenomenon and liver involvement (both $p<0.001$ ) and less articular involvement $(p=0.037)$ were observed among the EGMs in the ACA-positive group. Less frequency of leukopenia $(p=0.021)$, rheumatoid factor $(p<0.001)$, anti-Ro/SSA antibody positivity $(p<0.001)$, and hypergammaglobulinemia $(p=0.006)$, as well as higher positivity rates of anti-nuclear antibody and anti-topoisomerase antibody $(p<0.001$ and $p=0.006$, respectively) were found in the laboratory data in the ACA-positive pSS group.

Conclusions: Considering distinct phenotypes in hematological and serological features and EGMs, we should monitor the occurrence of these clinical features among pSS patients with ACA in caution.

Keywords: Sjogren's syndrome; Anticentromere antibody; Phenotype

\section{INTRODUCTION}

Primary Sjögren's syndrome $(\mathrm{pSS})$ is a slowly progressing autoimmune disease characterized by lymphocytic infiltration into the exocrine glands, resulting in dry eye and dry mouth [1]. Besides glandular dysfunction, pSS also induces various extra-glandular manifestations (EGMs), such as arthritis, Raynaud's phenomenon (RP), cytopenia, interstitial nephritis, and vasculitis [2]. Anti-Ro/SSA antibody is considered to play a crucial role in distinguishing the clinical features of this disease. Furthermore, since the announcement of American College of Rheumatology (ACR)/European League Against Rheumatism (EULAR) classification criteria in 2016, anti-Ro/ SSA antibody has emerged as the only disease-defining serologic marker [3]. However, $30 \%$ to $40 \%$ of pSS patients fulfilling the 2016 criteria do not present with this autoantibody [2]. No plausible hypotheses for how these patients show pSS-specific clinical features without the disease-specific marker have been suggested.

Several reports have described finding autoantibodies other than anti-Ro/SSA antibodies, such as anti- $\alpha$ fodrin, anti-carbonic anhydrase, and anti-type 3 muscarinic acetylcholine receptor antibodies in pSS patients [4]. Anticentromere antibody (ACA) was reported to be present in $3 \%$ to $10 \%$ of the pSS patients and has recent- ly been suggested as another clinically important marker in this disease [5-7]. Although several papers have been published on ACA-positive pSS, the results have been conflicting. Bournia et al. [5] reported ACA-positive pSS had a lower frequency of dry eyes, hypergammaglobulinemia, anti-Ro/SSA antibodies, and more frequent RP compared to ACA-negative pSS patients. Another study in Japan reported that this pSS subset showed lower focus scores and an increased amount of fibrous tissue in the minor salivary glands [8]. In contrast, a large cross-sectional study from the Sjögren's International Collaborative Clinical Alliance (SICCA) data presented opposing results on focus scores and the severity of glandular dysfunctions [9]. The most recent article from Notarstefano et al. [10] reported no significant difference in risk factors for lymphoproliferative diseases, such as germinal center-like lesions, between ACA-positive and ACA-negative pSS patients. According to a previous paper from a Korean group, an ACA-positive pSS group exhibited higher EULAR Sjögren's syndrome disease activity index (ESSDAI) scores [11]. Although the previous results could indicate that ACA affects pSS, inconsistency among the results makes it difficult to draw conclusions on the clinical impact of ACA on pSS phenotypes.

Discordance in the clinical results mentioned above could originate from relatively small sample sizes, the 
limited variety of clinical variables and heterogeneity of the study populations. Therefore, we aimed to clarify the clinical characteristics of pSS patients presenting with ACA positivity to overcome the limitations of past studies. We selected all the ACA-positive pSS patients from a Korean nationwide cohort for pSS to escalate the analysis power in the present study and compared them to the remaining ACA-negative pSS patients in all possible variables available in the cohort database.

\section{METHODS}

\section{Study population}

We extracted the study population from participants in the Korean Initiative of primary Sjögren's Syndrome (KISS) study. KISS is a nationwide prospective cohort database established to provide overall clinical data and samples of patients with pSS in Korea. Informed consent was obtained from all participants according to the principles of the Declaration of Helsinki. This study was approved by the Institutional Review Board of Seoul St. Mary's Hospital of the Catholic University of Korea (KC13ONMIo646). Recruitment began at Seoul St. Mary's Hospital, a tertiary care university-affiliated hospital and referral center in Seoul, Korea, in October 2013. By July 2017, the database included 501 patients with pSS from 11 other university hospitals across Korea, as well as Seoul St. Mary's Hospital. At the time of enrollment, all candidates had to fulfill the 2002 American-European Consensus Group (AECG) classification criteria [12] and/or the 2012 ACR criteria [13]. Any secondary cases possibly combined with other systemic autoimmune diseases, such as rheumatoid arthritis, systemic lupus erythematosus, and systemic sclerosis (SSc), were excluded from inclusion for the cohort by experienced rheumatologists' inspecting whether candidates fulfilled classification criteria for other connective tissue diseases. The additional exclusion criteria were radiation history to the head and neck area, chronic hepatitis $\mathrm{C}$ or human immunodeficiency virus infections, previous lymphoproliferative disease, sarcoidosis, graft-versus-host disease, amyloidosis, and immunoglobulin $\mathrm{G}$ (IgG) 4-related disease. We selected 318 patients who fulfilled the 2016 ACR/EULAR classification criteria [3] for pSS and had data on ACA positivity among all the par- ticipants of KISS study. Among them, 53 subjects were positive for ACA, while the remaining 265 were not.

\section{Assessment of glandular dysfunction}

We obtained all the clinical variables from the KISS database and used only baseline data acquired at the time of cohort enrollment in this study. According to the first and second items of the 2002 AECG classification criteria, positivity for subjective sicca symptoms was indicated [12]. As an objective test and a patient-reported symptom severity index for oral dryness, the unstimulated salivary flow (USF) rate and the xerostomia inventory (range, 11 to 55), self-checking questionnaire about severity for dry mouth, were evaluated [14]. The Schirmer I test, tear film break-up time, meibomian gland dysfunction, ocular staining scores (OSS) by both the SICCA method [15] and van Bijsterveld's system [16], and ocular surface disease index (range, o to 100) [17] were measured by ophthalmologists as objective tests related to ocular dryness. All ocular tests were performed for both eyes and worse results were selected for analysis. Minor salivary gland biopsies were obtained and evaluation of the degree of lymphocytic infiltration into the glands was conducted according to the SICCA protocol. The severity of the infiltration was presented as focus scores [18].

\section{Assessment of disease severity and extra-glandular manifestations}

Disease activity and long-term damage were evaluated by the ESSDAI [19] and the Sjögren's syndrome disease damage index (SSDDI) [20], respectively. These indices were calculated by two experienced rheumatologists. A visual analogue scale (VAS) was used for the patient and physician global assessment (range, o to $100 \mathrm{~mm}$ ) of disease activity. The EULAR Sjögren's syndrome patient-reported index (ESSPRI) was also assessed [21]. EuroQol (EQ)-5 dimensions (5D) time trade-off (TTO) values were derived from the South Korean reference data and EQ VAS scores were used to measure the patients' disease-related quality of life [22]. Information on EGMs presented earlier or at the time of enrollment for the subjects in each cohort was gathered. The definition of each type of EGM was based on a case report form from the KISS study and included (1) articular involvement, including a history or presence of morning stiffness over 30 minutes or any swollen or tender joints with 
the exclusion of degenerative arthritis; (2) RP, indicated by the presence of at least two color changes in distal phalanges including pallor, cyanosis, and redness combined with sensory changes, such as pain and tingling; (3) lymphadenopathy, defined as the presence of palpable lymph nodes over $1 \mathrm{~cm}$; (4) pulmonary involvement, including a history or presence of autoimmune-mediated or interstitial lung disease (ILD); (5) cutaneous vasculitis, indicated by the presence of palpable purpura suggesting the extravasation of red blood cells into the dermis; (6) kidney involvement, indicated by the presence of interstitial nephritis combined with renal tubular acidosis or glomerulonephritis combined with proteinuria over $0.5 \mathrm{~g} /$ day; (7) liver involvement, including the presence of autoimmune hepatitis (AIH) or autoimmune cholangitis or primary biliary cholangitis (PBC) diagnosed clinically or pathologically with the exclusion of toxic hepatitis and transient elevation of liver enzymes; (8) lymphoma, indicated by a history of lymphoma confirmed by pathology; (9) splenomegaly, defined as a palpable spleen below the left lower costal margin under full inspiration; (10) peripheral neuropathy, indicated by abnormal findings suggesting peripheral neuropathy in electroneurography; (11) myositis, the presence of myositis diagnosed by elevated serum muscle enzymes, such as creatinine kinase and abnormal findings suggesting myositis in electromyography or biopsy; (12) central nervous system involvement, demonstrated by the presence of cranial or optic neuritis or cerebrovascular accident or epilepsy; and (13) autoimmune thyroid disease, indicated by a history or presence of Graves' disease or Hashimoto's thyroiditis.

\section{Assessment of hematologic and serologic variables}

Cytopenia and hypergammaglobulinemia were defined according to the hematological and biological domains of ESSDAI, respectively [19]. Leukopenia was white blood cell counts $<4.00 \times 10^{3} / \mathrm{mm}^{3}$, neutropenia was neutrophils $<1.5 \times 10^{3} / \mathrm{mm}^{3}$, anemia was defined as a hemoglobin concentration $<12 \mathrm{~g} / \mathrm{dL}$, thrombocytopenia was platelet counts $<150 \times 10^{3} / \mathrm{mm}^{3}$, and hypergammaglobulinemia was defined as IgG levels $>16 \mathrm{~g} / \mathrm{L}$. Patients with cytopenia possibly caused by other medical conditions, such as nutrients deficiencies, drugs, or anemia of chronic disease, were excluded from the analysis. The presence of anti-nuclear antibodies (ANA) and rheuma- toid factor $(\mathrm{RF})$ was evaluated using an indirect immunofluorescence assay on HEp2 cells and immunoturbidimetric assay, respectively. A titer of 1:320 was used as a cut-off value to determine positivity for ANA, whereas values over $20 \mathrm{IU} / \mathrm{mL}$ were regarded as positive for $\mathrm{RF}$. All autoantibodies, as well as ACA were measured using commercial enzyme-linked immunosorbent assays (ELISA) without subtyping for each possible subset of autoantibodies.

\section{Statistical analysis}

Continuous variables were assessed about normal distribution using the Kolmogorov-Smirnov test. The non-normally distributed variables are expressed as medians and interquartile range (IQR) and were analyzed with the Mann-Whitney $U$ test. The chi-squared test and Fisher's exact test were used for categorical variables. IBM SPSS Statistics version 24.0 (IBM Corp., Armonk, NY, USA) was used for the statistical analysis. Statistical significance was considered at $p<0.05$.

\section{RESULTS}

\section{Basal demographics and exocrine secretion-related variables}

The median age of all selected subjects was 54 years (Table 1). As described in the methods, 53 of the 318 study patients were ACA-positive for a positivity rate of $16 \%$. The ACA-positive pSS patients (median, 56 years [IQR, 48 to 62$]$ ) were older than the ACA-negative patients (median, 54 years [IQR, 45 to 6o]; $p=0.042$ ). Disease duration was marginally longer in the ACA-negative group. Most patients were female (98.7\%) and had ocular and oral symptoms of dryness (98.1\% and $97.8 \%$, respectively). Although the frequency of USF positivity was not different between the two groups, the xerostomia inventory scores were higher in the ACA-positive group than in the ACA-negative group (median, 42 vs. 37), which meant that patients in the former group felt more symptoms of oral dryness than patients in the latter group did. Although the absolute values for the Schirmer I test and tear break-up time of the ACA-positive group were higher than those in the ACA-negative group $(p=0.038$ and $p=0.028$, respectively) among the ocular tests used to measure the severity of dry eyes, there were no dif- 
Table 1. Basal demographics and secretory function-related values

\begin{tabular}{|c|c|c|c|c|}
\hline Variable & $\begin{array}{c}\text { Total } \\
(\mathrm{n}=318)\end{array}$ & $\begin{array}{c}\mathrm{ACA}(+) \mathrm{pSS} \\
(\mathrm{n}=53)\end{array}$ & $\begin{array}{c}\operatorname{ACA}(-) p S S \\
(n=265)\end{array}$ & $p$ value \\
\hline Age, yr & $54(45-60)$ & $56(48-62)$ & $54(45-60)$ & 0.042 \\
\hline Disease duration, mon & $14(1-54)$ & $5(1-28)$ & $17(1-59)$ & 0.073 \\
\hline Female sex & $314(98.7)$ & $53(100)$ & $261(98.5)$ & $>0.999$ \\
\hline Symptoms for ocular dryness & $312(98.1)$ & $53(100)$ & $259(97.7)$ & 0.594 \\
\hline Symptoms for oral dryness & $311(97.8)$ & $52(98.1)$ & $259(97.7)$ & $>0.999$ \\
\hline \multicolumn{5}{|l|}{ Tests for ocular dryness } \\
\hline Schirmer I test, $<5 \mathrm{~mm} / 5 \mathrm{~min}$ & $242 / 298(81.2)$ & $36 / 44(81.8)$ & $206 / 254(81.1)$ & 0.911 \\
\hline Schirmer I test, $\mathrm{mm} / 5 \mathrm{~min}$ & $3(2-5)$ & $4(3-5)$ & $3(2-5)$ & 0.038 \\
\hline Tear break-up time, sec & $3(2-4)$ & $4(2-5)$ & $3(2-4)$ & 0.028 \\
\hline Meibomian gland dysfunction & $160 / 228(70.2)$ & $24 / 32(75 \cdot 0)$ & $136 / 196(69.4)$ & 0.520 \\
\hline OSS by SICCA method, $\geq 5$ (or Bijsterveld's method, $\geq 4$ ) & $105 / 223(47.1)$ & $12 / 32(37.5)$ & $93 / 191(48.7)$ & 0.240 \\
\hline \multicolumn{5}{|l|}{ Tests for oral dryness } \\
\hline Unstimulated salivary flow rate, $<0.1 \mathrm{~mL} / \mathrm{min}$ & $201 / 238(84.5)$ & $36 / 39(92.3)$ & $165 / 199(82.9)$ & 0.139 \\
\hline Unstimulated salivary flow rate, $\mathrm{mL} / 5 \mathrm{~min}$ & $0.3(0.0-1.2)$ & $0.3(0.1-0.5)$ & $0.3(0.0-1.2)$ & 0.086 \\
\hline \multicolumn{5}{|l|}{ Histopathology } \\
\hline Minor salivary gland biopsy positivity & $151 / 173(87 \cdot 3)$ & $35 / 37(94.6)$ & $116 / 136(85 \cdot 3)$ & 0.170 \\
\hline Focus score & $3(2-4)$ & $3(2-4)$ & $4(2-5)$ & 0.176 \\
\hline \multicolumn{5}{|l|}{ Patient-reported clinical indices } \\
\hline Xerostomia inventory & $38(30-43)$ & $42(34-46)$ & $37(30-43)$ & 0.040 \\
\hline Ocular surface disease index & $39(23-56)$ & $34(14-54)$ & $40(23-58)$ & 0.295 \\
\hline EULAR Sjögren's syndrome patient-reported index & $5.0(4.0-6.7)$ & $4.7(4.0-6.0)$ & $5 \cdot 3(4 \cdot 3-6.7)$ & 0.132 \\
\hline Pain & $3(0-5)$ & $2(0-5)$ & $3(0-5)$ & 0.041 \\
\hline Fatigue & $5(5-7)$ & $5(5-7)$ & $5(5-7)$ & 0.363 \\
\hline Dryness & $7(5-8)$ & $7(5-10)$ & $7(5-8)$ & 0.319 \\
\hline VAS for physician's global assessment & $30(17-45)$ & $33(10-45)$ & $30(17-45)$ & 0.770 \\
\hline VAS for patient's global assessment & $65(48-77)$ & $65(57-87)$ & $64(48-77)$ & 0.088 \\
\hline \multicolumn{5}{|l|}{ Quality-of-life-related indices } \\
\hline EuroQol VAS & $65(50-80)$ & $60(50-75)$ & $65(50-80)$ & 0.116 \\
\hline EuroQol-5 dimensions time trade-off value & $0.85(0.77-0.91)$ & $0.87(0.80-0.91)$ & $0.85(0.77-0.90)$ & 0.311 \\
\hline
\end{tabular}

Values are presented as median (interquartile range) or number (\%).

ACA, anticentromere antibody; pSS, primary Sjögren's syndrome; OSS, ocular staining score; SICCA, Sjögren's International Collaborative Clinical Alliance; EULAR, European League Against Rheumatism; VAS, visual analogue scale.

ferences in the proportion of positivity for Schirmer I test defined according to the 2016 ACR/EULAR criteria (regarded as positive if $<5 \mathrm{~mL}$ in 5 minutes). Differences in other ocular tests including the OSS, by both the SICCA method and van Bijsterveld's method, and the ocular surface damage index, were not significantly different between the two groups. The degree of lymphocytic in- filtration into the minor salivary glands represented by focus scores was also similar between the ACA-positive group and the ACA-negative group. The ratio of positive histopathologic findings in the exocrine glands, defined as focal lymphocytic sialadenitis according to the definition in the 2016 criteria, showed the same tendency in both groups. 
Table 2. EULAR Sjögren's syndrome disease activity index

\begin{tabular}{|c|c|c|c|c|}
\hline Variable & $\operatorname{Total}(\mathrm{n}=302)$ & $\operatorname{ACA}(+) \operatorname{pSS}(n=38)$ & $\operatorname{ACA}(-) \operatorname{pSS}(n=264)$ & $p$ value \\
\hline Total score & $3(1-6)$ & $2.5(0-6)$ & $3(1-6)$ & 0.668 \\
\hline Constitutional & $33(10.9)$ & $3(7 \cdot 9)$ & $30(11.4)$ & 0.781 \\
\hline Lymphadenopathy & $14(4.6)$ & $2(5 \cdot 3)$ & $12(4 \cdot 5)$ & 0.692 \\
\hline Glandular & $29(9.6)$ & $3(7 \cdot 9)$ & $26(9.8)$ & $>0.999$ \\
\hline Articular & $65(21.5)$ & $6(15.8)$ & $59(22.3)$ & 0.358 \\
\hline Cutaneous & $21(7.0)$ & o & $21(8.0)$ & 0.088 \\
\hline Pulmonary & $34(11.3)$ & $6(15.8)$ & $28(10.6)$ & 0.407 \\
\hline Renal & $4(1.3)$ & $1(2.6)$ & $3(1.1)$ & 0.418 \\
\hline Muscular & $1(0.3)$ & $\mathrm{o}$ & $1(0.4)$ & $>0.999$ \\
\hline Peripheral nervous system & $14(4 \cdot 6)$ & $3(7 \cdot 9)$ & $11(4.2)$ & 0.397 \\
\hline Central nervous system & $2(0.7)$ & 0 & $2(0.8)$ & $>0.999$ \\
\hline Hematological & $91(30.1)$ & $10(26.3)$ & $81(30.7)$ & 0.583 \\
\hline Biological & $168(55.6)$ & $16(42.1)$ & $152(57.6)$ & 0.073 \\
\hline
\end{tabular}

Values are presented as median (interquartile range) or number (\%).

EULAR, European League Against Rheumatism; ACA, anticentromere antibody; pSS, primary Sjögren's syndrome.

Table 3. Sjögren's syndrome disease damage index

\begin{tabular}{|c|c|c|c|c|}
\hline Variable & Total $(\mathrm{n}=302)$ & $\operatorname{ACA}(+) \operatorname{pSS}(n=38)$ & $\operatorname{ACA}(-) \operatorname{pSS}(n=264)$ & $p$ value \\
\hline Total score & $2(2-3)$ & $2(1-3)$ & $2(2-3)$ & 0.656 \\
\hline \multicolumn{5}{|l|}{ Ocular/salivary damage } \\
\hline Salivary flow impairment & $196(64.9)$ & $26(68.4)$ & $170(64.4)$ & 0.627 \\
\hline Loss of teeth & $30(9.9)$ & $7(18.4)$ & $23(8.7)$ & 0.078 \\
\hline \multicolumn{5}{|l|}{ Ocular damage } \\
\hline Tear flow impairment & $219(72.5)$ & $25(65.8)$ & $194(73 \cdot 5)$ & 0.320 \\
\hline Structural abnormalities & $194(64.2)$ & $22(57.9)$ & $172(65.2)$ & 0.383 \\
\hline \multicolumn{5}{|l|}{ Neurologic damage } \\
\hline Central nervous system involvement & $2(0.7)$ & o & $2(0.8)$ & $>0.999$ \\
\hline Peripheral neuropathy & $11(3.6)$ & $2(5 \cdot 3)$ & $9(3.4)$ & 0.635 \\
\hline Pleuropulmonary damage & $6(2.0)$ & $\mathrm{O}$ & $6(2.3)$ & $>0.999$ \\
\hline Renal impairment & $5(1.4)$ & 0 & $5(1.9)$ & $>0.999$ \\
\hline Lymphoproliferative disease & o & o & o & \\
\hline
\end{tabular}

Values are presented as median (interquartile range) or number (\%).

ACA, anticentromere antibody; pSS, primary Sjögren's syndrome.

\section{Patient-reported clinical indices and quality-of-life- related indices}

VAS scores for disease activity evaluated by each physician and patient were not significantly different between the two groups, as shown in Table 1. The VAS scores in the ACA-positive group were lower than those in the
ACA-negative group in the ESSPRI pain domain $(p=$ 0.041), whereas the overall scores in the index showed no inter-group differences. EQ-5D TTO and EQ VAS scores, which could reflect the patients' disease-related quality of life, were not significantly different between the two groups. 


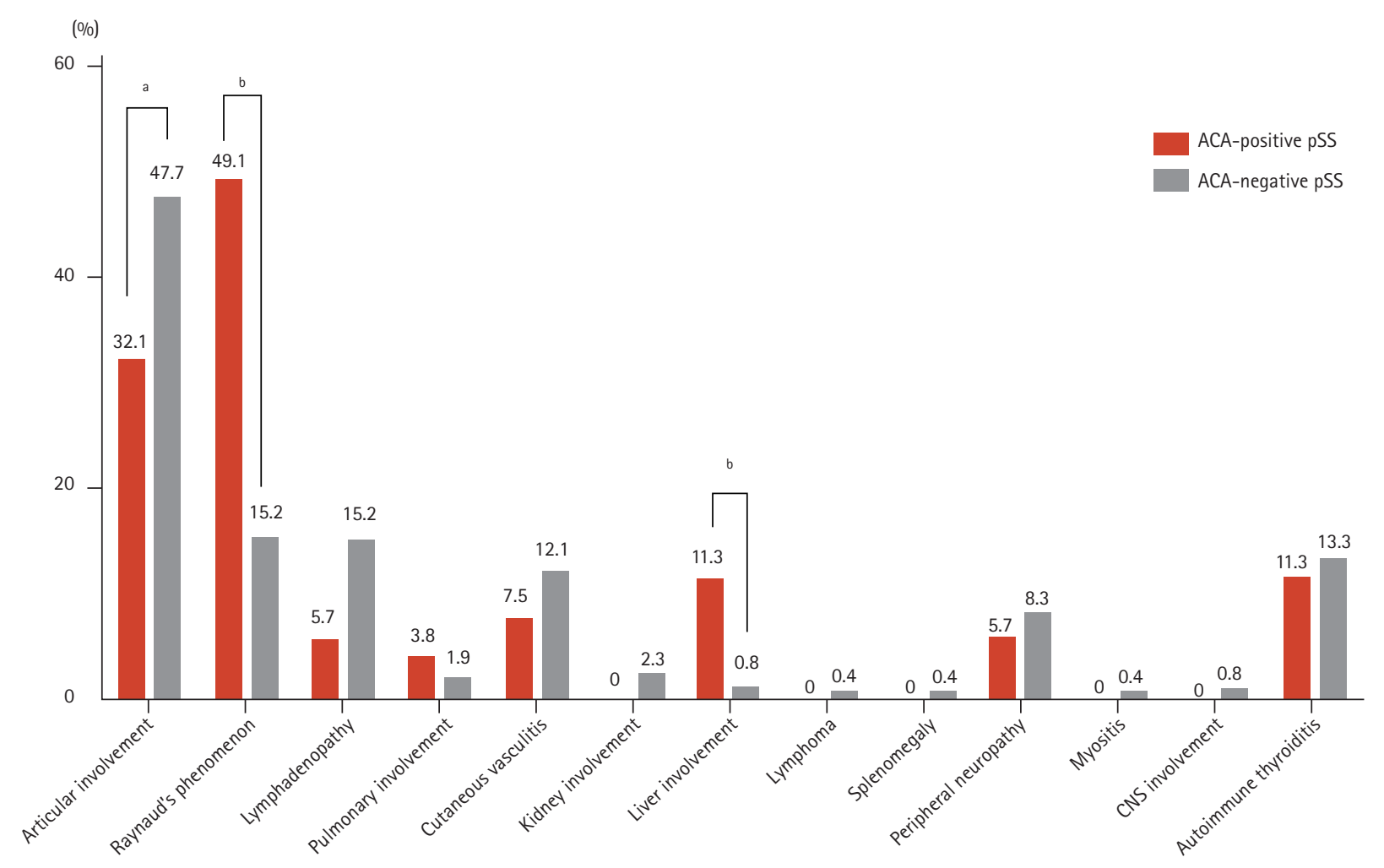

Figure 1. Extra-articular manifestations of patients with anticentromere antibody-positive primary Sjögren's syndrome and patients with anticentromere antibody-negative primary Sjögren's syndrome. All figures mean percentages. ACA, anticentromere antibody; pSS, primary Sjögren's syndrome; CNS, central nervous system. ${ }^{a} p<0.05,{ }^{b} p<0.001$.

\section{Disease activity and damage-related index}

The overall scores for both the ESSDAI and SSDDI (Tables 2 and 3, respectively), which represent the degree of disease activity and disease-associated long-term damage, respectively, were not statistically different between the two groups. We also compared the frequencies of positivity for each domain of the ESSDAI and SSDDI. The status of ACA positivity did not make inter-group differences in each domain of both indices.

\section{Extra-glandular manifestations}

Among all EGMs, articular involvement, including arthralgia and arthritis, occurred less (32.1\% vs. 47.7\%, $p=0.037$ ), whereas liver involvement (11.3\% vs. $0.8 \%$, $p<0.001)$ and RP (49.1\% vs. $15.2 \%, p<0.001)$ presented more frequently in the ACA-positive group (Fig. 1). Among the six patients with liver involvement in the ACA-positive group, three had PBC, one had AIH, and the other two patients had both PBC and AIH. All the patients with liver involvement described above were diagnosed by pathologic confirmation. Although there was no statistical significance, seven cases of pulmonary involvement were observed in both study groups (two in the ACA-positive group while five in the ACA-negative group). All pulmonary involvements were confirmed as ILD by using computed tomography. A radiological type of ILD in all pulmonary involvements was presented as 'nonspecific interstitial pneumonia.'

\section{Hematological and serological features}

The ACA-positive group presented a lower prevalence of leukopenia compared to the ACA-negative group (17.3\% vs. $33.5 \%, p=0.021$ ), whereas the absolute white blood cell count was not statistically different between the two groups, as shown in Table 4. The median hemoglobin concentration of the ACA-positive group was higher than that in the other group $(p=0.043)$, but the proportion of patients with anemia was not different significantly. Nearly all patients in the ACA-positive group showed ANA-positivity, whereas RF was less like- 
Table 4. Laboratory data

\begin{tabular}{|c|c|c|c|c|}
\hline Variable & Total $(\mathrm{n}=315)$ & $\mathrm{ACA}(+) \mathrm{pSS}(\mathrm{n}=52)$ & $\operatorname{ACA}(-) \operatorname{pSS}(n=263)$ & $p$ value \\
\hline \multicolumn{5}{|l|}{ Hematologic tests } \\
\hline White blood cell, $\times 10^{3} / \mathrm{mm}^{3}$ & $4.590(3.740-5.600)$ & $4.940(4.155-5.717)$ & $4.520(3.680-5.570)$ & 0.097 \\
\hline Hemoglobin, g/dL & $12.9(12.0-13.7)$ & $13.2(12.4-13.7)$ & $12.8(12.0-13.6)$ & 0.043 \\
\hline Platelet, $\times 10^{3} / \mathrm{mm}^{3}$ & $221(189-256)$ & $205(186-253)$ & $221(190-257)$ & 0.325 \\
\hline Leukopenia, $<4.00 \times 10^{3} / \mathrm{mm}^{3}$ & $97(30.8)$ & $9(17 \cdot 3)$ & $88(33 \cdot 5)$ & 0.021 \\
\hline Anemia, $<12 \mathrm{~g} / \mathrm{dL}$ & $70(22.2)$ & $7(13.5)$ & $63(24.0)$ & 0.096 \\
\hline Thrombocytopenia, $<150 \times 10^{3} / \mathrm{mm}^{3}$ & $23(7 \cdot 3)$ & $3(5.8)$ & $20(7 \cdot 6)$ & 0.778 \\
\hline \multicolumn{5}{|l|}{ Serologic tests } \\
\hline Immunoglobulin G, mg/dL & $1,552(1,337-1,948)$ & $1,361(1,129-1,700)$ & $1,611(1,377-1,972)$ & $<0.001$ \\
\hline Immunoglobulin A, mg/dL & $265(195-366)$ & $249(167-383)$ & $267(206-366)$ & 0.356 \\
\hline Immunoglobulin $\mathrm{M}, \mathrm{mg} / \mathrm{dL}$ & $118(86-154)$ & $144(108-193)$ & $114(83-149)$ & $<0.001$ \\
\hline Hypergammaglobulinemia & $145 / 309(46.9)$ & $15 / 51(29.4)$ & $130 / 258(50.4)$ & 0.006 \\
\hline $\mathrm{C}_{3}, \mathrm{mg} / \mathrm{dL}$ & $93(82-102)$ & $88(80-99)$ & $93(82-103)$ & 0.244 \\
\hline $\mathrm{C}_{4}, \mathrm{mg} / \mathrm{dL}$ & $22(18-26)$ & $21(18-25)$ & $22(18-26)$ & 0.336 \\
\hline Low $\mathrm{C}_{3},<76 \mathrm{mg} / \mathrm{dL}$ & $50 / 310(16.1)$ & $9(17 \cdot 3)$ & $41 / 258(15 \cdot 9)$ & 0.800 \\
\hline Low $\mathrm{C}_{4},<12 \mathrm{mg} / \mathrm{dL}$ & $15 / 310(4.8)$ & $2(3.8)$ & $13 / 258(5.0)$ & $>0.999$ \\
\hline Cryoglobulin & $5 / 292(1.7)$ & o & $5(1.9)$ & $>0.999$ \\
\hline \multicolumn{5}{|l|}{ Tests about autoantibodies } \\
\hline Rheumatoid factor positivity, > $20 \mathrm{IU} / \mathrm{mL}$ & $197 / 306(64.4)$ & $20(38.5)$ & $177 / 254(69.7)$ & $<0.001$ \\
\hline Anti-cyclic citrullinated peptides antibody, U/mL & $1.4(0.8-4.0)$ & $1.3(0.5-2.6)$ & $1.4(0.9-4.5)$ & 0.053 \\
\hline Anti-nuclear antibody positivity, titer $\geq 1: 320$ & $197 / 303(65.0)$ & $49 / 51(96.1)$ & $148 / 252(58.7)$ & $<0.001$ \\
\hline Anti-DNA antibody positivity & $10 / 306(3.3)$ & $2 / 46(4 \cdot 3)$ & $8 / 260(3.1)$ & 0.65 \\
\hline Anti-Ro/SSA antibody positivity & $283(89.8)$ & $32(61.5)$ & $251(95 \cdot 4)$ & $<0.001$ \\
\hline Anti-La/SSB antibody positivity & $161 / 314(51.3)$ & $16 / 50(32.0)$ & $145(55 \cdot 1)$ & 0.003 \\
\hline Anti-topoisomerase antibody positivity & $6 / 294(2.0)$ & $4 / 46(8.7)$ & $2 / 248(0.8)$ & 0.006 \\
\hline Anti-ribonucleoprotein antibody positivity & $6 / 206(2.9)$ & $2 / 38(5 \cdot 3)$ & $4 / 168(2.4)$ & 0.306 \\
\hline Anti-Jo-1 antibody positivity & $3 / 304(1.0)$ & o & $3(1.1)$ & $>0.999$ \\
\hline
\end{tabular}

Values are presented as median (interquartile range) or number (\%).

ACA, anticentromere antibody; pSS, primary Sjögren's syndrome.

ly to be present in this group (both $p<0.001$ ). Both the frequency of hypergammaglobulinemia and the median of IgG level were lower in the ACA-positive group $(p=$ 0.006 and $p<0.001$, respectively), which could indicate a relatively lower activation of B-cells compared to the ACA-negative group. Among all the extractable nuclear autoantibodies that could have been evaluated in our study, anti-Ro/SSA antibodies, anti-La/SSB antibodies, and anti-topoisomerase antibodies showed statistically significant disparities between the two groups. The presence of anti-Ro/SSA antibodies and anti-La/SSB an- tibodies was significantly less frequent $(p<0.001$ and $p=$ 0.003 , respectively), whereas the anti-topoisomerase antibody was present more frequently in the ACA-positive group $(p=0.006)$.

\section{DISCUSSION}

ACA has been regarded as a specific autoantibody mainly associated with SSc, especially in a subset of patients showing limited skin involvement. It also has been 
found in patients with systemic lupus erythematosus, rheumatoid arthritis, PBC, and pSS. For the recent decade, ACA has been suggested as a possible marker relevant to pSS because of its consistent detection in sera of pSS patients. Therefore, we compared various clinical aspects of the ACA-positive pSS patients and ACA-negative patients available in our prospective cohort database to characterize the ACA-positive subsets in the present study. Patients in the ACA-positive group were older, had more frequent EGMs, such as RP and liver involvement, less frequent leukopenia and hypergammaglobulinemia, and a lower positive ratio of anti-Ro/ SSA antibodies. They showed similar severities in glandular dysfunction, disease activity, and long-term damage compared to the ACA-negative group.

Despite prevalence of ACA seropositivity in pSS, mechanisms and influence of ACA on pathogenesis of pSS have been scarcely revealed to date. A recent study reported local production of ACA from antibody-secreting cells in lesions of ACA-positive pSS patients [23]. However, questions about why these antibodies are made from antibody-secreting cells and whether they directly influence on the inflammation of salivary glands are still unsolved. In addition to immunologic problems, clinical aspects of ACA-positive pSS also remain some issues to be addressed.

The results showing that ACA-positive pSS were older agreed with previous reports, including the former Korean study data and a larger study in other regions $[8,9,11,24]$. However, in terms of severity in secretory dysfunctions of the exocrine glands, the results of the present study disagreed with the largest-scaled study results from the SICCA cohort. Baer et al. [9] reported that more severe exocrine glandular dysfunction, demonstrated by USF, Schirmer's I test, and focus scores, was observed in ACA-positive pSS patients. Our study did not show more severe glandular dysfunction in ACA-positive pSS patients, except the xerostomia inventory. Rather, ACA-negative pSS patients showed lower absolute values in ocular test, such as Schirmer I test and tear film break-up time. Although there were more study participants in the other study, such disparity could originate from the unique characteristics of a specific ethnicity because the anti-Ro/SSA antibody status, regarded as a more disease-indicative marker of pSS, also had little association with glandular dysfunction in Korean pa- tients, as reported in our previously published paper [25]. The tendency toward no difference in glandular dysfunction, regardless of ACA positivity, was also observed in smaller epidemiologic studies in adjacent regions $[8,11,24]$. We could assume that ACA had a limited impact on the severity of secretory dysfunctions of the exocrine glands in Korean patients in this study.

The frequency of several types of EGMs, including articular involvement, RP, and liver involvement, were different depending on the presence of ACAs, whereas all the indices that indicated disease activity, disease-related long-term damage, and quality of life, were not. Lee et al. [11] suggested that ACA-positive pSS patients had higher ESSDAI scores compared to ACA-negative pSS patients in Korea. In our study, which included a larger SS population in Korea than the former study, ESSDAI scores, including all the scores of each subdomain, as well as the overall scores, showed no disparity between the two groups. We suggest that ACAs have little clinical impact in terms of systemic activity in pSS, similar to the glandular dysfunction findings. Higher RP frequency among the EGMs seen in this study was consistent with several previous studies [5,7,8,11,26]. Well-known clinical correlations between ACA and RP frequency in limited types of SSc make this finding reasonably acceptable. Our novel EGM findings indicated the clinical influence of ACA on the frequency of articular and liver involvement. Less frequent articular involvement, including both arthralgia and arthritis in ACA-positive pSS patients, could be a result of a lower RF positivity in that pSS subset. The discrepancy between the EGMs and the articular domain of the ESSDAI results could originate from the degree of stringency of the two parameters. Considering similar levels of anti-cyclic citrullinated peptide antibody from the sera of patients in both study groups and our definition of articular involvement in EGMs, the articular symptoms in ACA-positive pSS patients might be subclinical rather than overt arthritis. The higher prevalence of liver involvement in the ACA-positive group was observed mainly in the form of PBC. ACAs were reported in about $15 \%$ of the sera of patients with $\mathrm{PBC}$ and were related to worse outcomes [27]. While the prevalence of pSS was suggested to be between $20 \%$ and $40 \%$ among patients with PBC [28], PBC occurred less than $2 \%$ among pSS patients [29,30]. Although anti-mitochondrial antibody is known to be 
more specific for PBC, ACA-positive pSS patients presented with a higher prevalence of PBC, which was 10\% in the present study. This suggests that the disparity in the frequency of liver involvement between the two groups might originate from the clinical associations of $\mathrm{ACA}$ and PBC.

In hematological features, less prevalent leukopenia and higher hemoglobin concentrations seen in the current study were also reported in other studies $[8,31]$. Whether there was statistical significance or not, pSS patients with ACA had consistently higher whole blood cell counts compared to patients without the autoantibody. The IgG blood levels were also lower in the ACA-positive pSS group, implicating a lower degree of chronic B-cell activation. Less frequent anti-Ro/SSA antibody and anti-La/SSB antibody positivity in association with the presence of ACA are well-known features of ACA-positive pSS patients [5,9]. Unfortunately, our KISS database lacked the information about the subtypes of anti-Ro/SSA antibodies. A previous study reported that some commercial ELISA kits only detect anti-Ro6o antibodies [32]. Therefore, there could be some possibilities to miss seropositivity for anti-Ro52 antibodies, which are known to be also associated with pSS-specific clinical features, in anti-Ro/ SSA-negative patients. Although lower degrees of chronic B-cell activation and the prevalence of cytopenia could suggest lower risks for the development of lymphoma in ACA-positive pSS patients, we were not able to confirm this in our study because follow-up data was not included in the present study. Even if included, an extremely low incidence of lymphoma in the KISS study patients was observed during the follow-up periods. Future studies should be conducted to investigate this query.

The present study included high-quality data derived from a prospective cohort of uniformly classified participants under strict inclusion and exclusion criteria across an entire nation. This enabled the included patients to be homogeneous and the authors to explore genuine clinical impacts of ACA on pSS phenotypes. Various clinical variables related to $\mathrm{SS}$, including glandular dysfunctions, extra-glandular symptoms and laboratory values, were analyzed. Despite such strengths of this study, some limitations also exist. First, among total 501 subjects in the KISS cohort, 318 patients involving information on serological status for ACA were selected as study subjects in the present study. Because only a part of whole popula- tion in the cohort was included, there could be a selection bias which results in decreased representativeness of the study subjects. Second, because the KISS study was designed to investigate pSS and not SSc, separate information related to SSc, such as presence of sclerodactyly or telangiectasia and nailfold capillary microscopic findings were lacking in the database. Therefore, even if patients with SSc fulfilling the 2013 ACR/EULAR criteria for SSc were excluded in each center at the time of enrollment, we could not compare other relevant clinical features possibly observed in ACA-positive patients. Third, patients with an early phase of SSc might not fulfill the 2013 classification criteria for SSc at the time of enrollment for the cohort. Because of cross-sectional design of the present study, incidence of new SSc cases is not reflected here. Subsequent studies regarding the incidence of SSc among ACA-positive patients during follow-up periods could bring us additional valuable information. Lastly, ACA is known to recognize three species, centromere protein (CENP)-A, CENP-B, and CENP-C. Autoantibodies in sera from patients with pSS mainly bind only CENP-C, while those from SSc patients bind CENP-B and CENP-C [33,34]. In our study, we used a commercial ELISA kit to detect ACAs that recognizes all species of ACAs without subtyping. Therefore, ACA species prevalence could not be observed in the present study. Addressing these unmet findings in future studies could suggest additional clues for pathophysiological mechanisms of ACA-positive pSS.

In conclusion, ACA positivity had little clinical impact on the severity of glandular dysfunction and systemic activity in pSS patients but was related to lower B-cell expansion markers and a higher prevalence of EGMs related to features of other ACA-specific autoimmune diseases, such as SSc and PBC. Although there were no cases with lymphoma and full-blown SSc at the time of baseline, it would be worthwhile to monitor for these diseases in the ACA-positive pSS subset in a future study.

\section{KEY MESSAGE}

1. Anticentromere antibody makes distinguishing influences on clinical phenotypes in primary Sjögren's syndrome.

2. The positivity for anticentromere antibody has a 
little impact on glandular dysfunctions and systemic disease activity.

3. Extra-glandular manifestations related to systemic sclerosis are more frequent in anticentromere antibody-positive Sjögren's syndrome.

\section{Conflict of interest}

No potential conflict of interest relevant to this article was reported.

\section{Acknowledgments}

This study was supported by a grant from the Korean Health Technology R\&D Project, Ministry of Health and Welfare, Republic of Korea ( $\left.\mathrm{HI}_{13} \mathrm{CoO} 16\right)$.

\section{REFERENCES}

1. Fox RI. Sjogren's syndrome. Lancet 2005;366:321-331.

2. Brito-Zeron P, Baldini C, Bootsma H, et al. Sjogren syndrome. Nat Rev Dis Primers 2016;2:16047.

3. Shiboski CH, Shiboski SC, Seror R, et al. 2016 American College of Rheumatology/European League Against Rheumatism classification criteria for primary Sjogren's syndrome: a consensus and data-driven methodology involving three international patient cohorts. Arthritis Rheumatol 2017;69:35-45.

4. Bournia VK, Vlachoyiannopoulos PG. Subgroups of Sjogren syndrome patients according to serological profiles. J Autoimmun 2012;39:15-26.

5. Bournia VK, Diamanti KD, Vlachoyiannopoulos PG, Moutsopoulos HM. Anticentromere antibody positive Sjogren's syndrome: a retrospective descriptive analysis. Arthritis Res Ther 2010;12:R47.

6. Nakamura H, Kawakami A, Hayashi T, et al. Anti-centromere antibody-seropositive Sjogren's syndrome differs from conventional subgroup in clinical and pathological study. BMC Musculoskelet Disord 2010;11:140.

7. Ramos-Casals M, Nardi N, Brito-Zeron P, et al. Atypical autoantibodies in patients with primary Sjogren syndrome: clinical characteristics and follow-up of 82 cases. Semin Arthritis Rheum 2006;35:312-321.

8. Katano K, Kawano M, Koni I, Sugai S, Muro Y. Clinical and laboratory features of anticentromere antibody positive primary Sjogren's syndrome. J Rheumatol 2001;28:2238-2244.

9. Baer AN, Medrano L, McAdams-DeMarco M, Gniadek TJ. Association of anticentromere antibodies with more severe exocrine glandular dysfunction in Sjogren's syndrome: analysis of the Sjogren's International Collaborative Clinical Alliance Cohort. Arthritis Care Res (Hoboken) 2016;68:1554-1559.

10. Notarstefano C, Croia C, Pontarini E, et al. A clinical and histopathological analysis of the anti-centromere antibody positive subset of primary Sjogren's syndrome. Clin Exp Rheumatol 2018;36 Suppl 112:145-149.

11. Lee KE, Kang JH, Lee JW, et al. Anti-centromere antibody-positive Sjogren's syndrome: a distinct clinical subgroup? Int J Rheum Dis 2015;18:776-782.

12. Vitali C, Bombardieri S, Jonsson R, et al. Classification criteria for Sjogren's syndrome: a revised version of the European criteria proposed by the American-European Consensus Group. Ann Rheum Dis 2002;61:554-558.

13. Shiboski SC, Shiboski CH, Criswell L, et al. American College of Rheumatology classification criteria for Sjogren's syndrome: a data-driven, expert consensus approach in the Sjogren's International Collaborative Clinical Alliance cohort. Arthritis Care Res (Hoboken) 2012;64:475-487.

14. Thomson WM, Chalmers JM, Spencer AJ, Williams SM. The Xerostomia Inventory: a multi-item approach to measuring dry mouth. Community Dent Health 1999;16:12-17.

15. Whitcher JP, Shiboski CH, Shiboski SC, et al. A simplified quantitative method for assessing keratoconjunctivitis sicca from the Sjogren's Syndrome International Registry. Am J Ophthalmol 2010;149:405-415.

16. van Bijsterveld OP. Diagnostic tests in the Sicca syndrome. Arch Ophthalmol 1969;82:10-14.

17. Schiffman RM, Christianson MD, Jacobsen G, Hirsch JD, Reis BL. Reliability and validity of the Ocular Surface Disease Index. Arch Ophthalmol 2000;118:615-621.

18. Daniels TE, Cox D, Shiboski CH, et al. Associations between salivary gland histopathologic diagnoses and phenotypic features of Sjogren's syndrome among 1,726 registry participants. Arthritis Rheum 2011;63:2021-2030.

19. Seror R, Bowman SJ, Brito-Zeron P, et al. EULAR Sjogren's syndrome disease activity index (ESSDAI): a user guide. RMD Open 2015;1:e0ooo22.

20. Vitali C, Palombi G, Baldini C, et al. Sjogren's Syndrome Disease Damage Index and Disease Activity Index: scor- 
ing systems for the assessment of disease damage and disease activity in Sjogren's syndrome, derived from an analysis of a cohort of Italian patients. Arthritis Rheum 2007;56:2223-2231.

21. Seror R, Ravaud P, Mariette X, et al. EULAR Sjogren's Syndrome Patient Reported Index (ESSPRI): development of a consensus patient index for primary Sjogren's syndrome. Ann Rheum Dis 2011;70:968-972.

22. Lee YK, Nam HS, Chuang LH, et al. South Korean time trade-off values for EQ-5D health states: modeling with observed values for 101 health states. Value Health 2009;12:1187-1193.

23. Takeshita M, Suzuki K, Kaneda Y, et al. Antigen-driven selection of antibodies against SSA, SSB and the centromere 'complex', including a novel antigen, MIS12 complex, in human salivary glands. Ann Rheum Dis 2020;79:150-158.

24. Suzuki Y, Fujii H, Nomura H, et al. Impact of double positive for anti-centromere and anti-SS-a/Ro antibodies on clinicopathological characteristics of primary Sjogren's syndrome: a retrospective cohort study. Mod Rheumatol 2018;28:872-878.

25. Park Y, Lee J, Koh JH, et al. Distinct clinical characteristics of anti-Ro/SSA-negative primary Sjogren's syndrome: data from a nationwide cohort for Sjogren's syndrome in Korea. Clin Exp Rheumatol 2019;37 Suppl 118:107-113.

26. Tsukamoto M, Suzuki K, Takeuchi T. Clinical and immunological features of anti-centromere antibody-positive primary Sjogren's syndrome. Rheumatol Ther 2018;5:499505 .

27. Yang WH, Yu JH, Nakajima A, Neuberg D, Lindor K, Bloch
DB. Do antinuclear antibodies in primary biliary cirrhosis patients identify increased risk for liver failure? Clin Gastroenterol Hepatol 2004;2:1116-1122.

28. Tsianos EV, Hoofnagle JH, Fox PC, et al. Sjogren's syndrome in patients with primary biliary cirrhosis. Hepatology 1990;11:730-734.

29. Montano-Loza AJ, Crispin-Acuna JC, Remes-Troche JM, Uribe M. Abnormal hepatic biochemistries and clinical liver disease in patients with primary Sjogren's syndrome. Ann Hepatol 2007;6:150-155.

30. Malladi AS, Sack KE, Shiboski SC, et al. Primary Sjogren's syndrome as a systemic disease: a study of participants enrolled in an international Sjogren's syndrome registry. Arthritis Care Res (Hoboken) 2012;64:911-918.

31. Caramaschi P, Biasi D, Carletto A, et al. Sjogren's syndrome with anticentromere antibodies. Rev Rhum Engl Ed 1997;64:785-788.

32. Peene I, Meheus L, Veys EM, De Keyser F. Diagnostic associations in a large and consecutively identified population positive for anti-SSA and/or anti-SSB: the range of associated diseases differs according to the detailed serotype. Ann Rheum Dis 2002;61:1090-1094.

33. Gelber AC, Pillemer SR, Baum BJ, et al. Distinct recognition of antibodies to centromere proteins in primary Sjogren's syndrome compared with limited scleroderma. Ann Rheum Dis 2006;65:1028-1032.

34. Tanaka N, Muro Y, Suzuki Y, et al. Anticentromere antibody-positive primary Sjogren's syndrome: epitope analysis of a subset of anticentromere antibody-positive patients. Mod Rheumatol 2017;27:115-121. 